\title{
Home medicines reviews following acute coronary syndrome: study protocol for a randomized controlled trial
}

Daniel DL Bernal ${ }^{1 *}$, Leanne Stafford ${ }^{1}$, Luke RE Bereznicki ${ }^{1}$, Ronald L Castelino ${ }^{1}$, Patricia M Davidson ${ }^{2}$ and Gregory M Peterson ${ }^{1}$

\begin{abstract}
Background: Despite continual improvements in the management of acute coronary syndromes, adherence to guideline-based medications remains suboptimal. We aim to improve adherence with guideline-based therapy following acute coronary syndrome using an existing service that is provided by specifically trained pharmacists, called a Home Medicines Review. We have made two minor adjustments to target the focus of the existing service including an acute coronary syndrome specific referral letter and a training package for the pharmacists providing the service.
\end{abstract}

Methods/Design: We will be conducting a randomized controlled trial to compare the directed home medicines review service to usual care following acute coronary syndromes. All patients aged 18 to 80 years and with a working diagnosis of acute coronary syndrome, who are admitted to two public, acute care hospitals, will be screened for enrolment into the trial. Exclusion criteria will include: not being discharged home, documented cognitive decline, non-Medicare eligibility, and presence of a terminal malignancy. Randomization concealment and sequence generation will occur through a centrally-monitored computer program. Patients randomized to the control group will receive usual post-discharge care. Patients randomized to receive the intervention will be offered usual post-discharge care and a directed home medicines review at two months post-discharge. The study endpoints will be six and twelve months post-discharge. The primary outcome will be the proportion of patients who are adherent to a complete, guideline-based medication regimen. Secondary outcomes will include hospital readmission rates, length of hospital stays, changes in quality of life, smoking cessation rates, cardiac rehabilitation completion rates, and mortality.

Discussion: As the trial is closely based on an existing service, any improvements observed should be highly translatable into regular practice. Possible limitations to the success of the trial intervention include general practitioner approval of the intervention, general practitioner acceptance of pharmacists' recommendations, and pharmacists' ability to make appropriate recommendations. A detailed monitoring process will detect any barriers to the success of the trial. Given that poor medication persistence following acute coronary syndrome is a worldwide problem, the findings of our study may have international implications for the care of this patient group.

Trial registration: Australian New Zealand Clinical Trials Registry ACTRN12611000452998

Keywords: Acute coronary syndrome, Home medicines review, Medication adherence

\footnotetext{
* Correspondence: ddbernal@utas.edu.au

'Unit for Medication Outcomes Research and Education (UMORE), School of Pharmacy, University of Tasmania (UTAS), Sandy Bay Campus, Tasmania 7001, Australia

Full list of author information is available at the end of the article
}

\section{() Biomed Central}

(C) 2012 Bernal et al; licensee BioMed Central Ltd. This is an Open Access article distributed under the terms of the Creative Commons Attribution License (http://creativecommons.org/licenses/by/2.0), which permits unrestricted use, distribution, and reproduction in any medium, provided the original work is properly cited. 


\section{Background}

Optimal medication management is critical to improving outcomes following acute coronary syndrome (ACS), yet prescribing practices and patient behaviors often fall below recognized targets [1-3]. Research demonstrates that the prescribing of guideline-based medications following ACS, including antithrombotics, beta-blockers, angiotensin-converting enzyme inhibitors, and statins, has improved over recent years [4] but is not yet ideal [3]. When patients with ACS leave hospital and return to the community, however, a more significant problem becomes apparent due to the premature discontinuation of guideline-based therapies by patients and/or clinicians [1-3]. Furthermore, the majority of the morbidity and mortality that occurs in the early post-discharge period can be attributed to a failure to appropriately use these existing medications [5-8]. For example, premature medication discontinuation, when measured at one month post-discharge in a myocardial infarction population, decreased 12-month survival by $8 \%$ [2]. While, understandably, much of the focus on improving patient outcomes following ACS has centered on the acute phase of care, it is crucial that more attention is paid to the development of new, post-discharge strategies to improve outcomes following hospital discharge.

Similar to other developed countries [2], poor medication persistence following ACS has been recognized as an unresolved problem in Australia [3,9]. A recent Australia-wide quality improvement study, called Discharge Management of Acute Coronary Syndrome (DMACS) utilized an education-based intervention to improve inhospital, guideline-based prescribing and this resulted in a significant improvement in the proportion of patients prescribed four guideline-based medications at discharge, from $57 \%$ at baseline to $69 \%$ post-intervention $(P<0.0001)$ [3]. A telephone follow-up of the postintervention group, however, revealed a $17 \%$ decline in the proportion of patients taking all four guidelinebased medications at three months post-discharge. This equated to a $66 \%$ loss of the improvement in prescribing that was recorded at discharge, by just three months post-discharge and suggested that the intervention was effective at improving in-hospital prescribing, but there was little effect on long-term medication use. The authors noted in their discussion that further community follow-up once the patients were settled in their homes could improve the poor rates of post-discharge medication persistence.

\section{ACS post-discharge services}

Cardiac rehabilitation has been continually shown to improve multiple outcomes following ACS, including mortality [10]. As such, the DMACS study also aimed to improve referral rates and uptake of cardiac rehabilitation. While post-intervention referral rates significantly increased from $67 \%$ to $73 \%(P=0.001)$, attendance rates remained unchanged at just 33\% [3], which is in line with the low worldwide attendance rates for cardiac rehabilitation programs [11]. A Cochrane review has highlighted the potential for structured interventions to successfully increase cardiac rehabilitation uptake; however, interventions aimed at improving cardiac rehabilitation completion rates have generally proven less successful [12]. Although the benefits of cardiac rehabilitation are well-established, attendance and completion rates remain low, and evidence-based strategies to overcome these problems and increase access to the service are yet to be recognized.

Cardiac rehabilitation programs are designed to increase adherence to both pharmacological and nonpharmacological therapies [13]. However, there have been few evaluations of the specific effect of these programs on medication adherence. One study of a relatively high-intensity cardiac rehabilitation program was able to demonstrate an improvement in medication adherence at three months post-discharge [14], but despite these initial improvements, the benefit was short-lived, with both treatment and control groups reporting the same level of medication adherence at six months post-discharge. There is a clear need for a more intensive multidisciplinary approach to the post-discharge management of ACS, including interventions specifically directed at improving medication management. These interventions should be designed to both complement and promote the existing cardiac rehabilitation programs, as well as being accessible to the large majority of patients who currently find reasons not to attend cardiac rehabilitation.

The Home Medicines Review (HMR) program is an existing community-based service that has the potential to be tailored to meet the needs of patients recently discharged from hospital following ACS. Although this potential has not been fully realized in the past [15], HMRs may be used to particularly target patients' persistence and adherence to guideline-based medications following ACS. The HMR service currently involves general practitioner (GP) referral of patients to an HMR-accredited pharmacist, often through a community pharmacist liaison [16]. The accredited pharmacist will visit the patient in their home, discuss their medication taking habits, and provide education or adherence interventions where required. Following the home visit the accredited pharmacist writes a report for the GP noting their observations and any clinical adjustments that could be made to the patient's medication regimen. Based on this report, the GP is expected to complete an 
agreed management plan, selecting the accredited pharmacist's recommendations which they agree to implement and/or follow up. The service is currently available in Australia, free of charge to Australian citizens under funding arrangements through the public health system, Medicare Australia. The GP, accredited pharmacist, and community pharmacist involved with an HMR service are paid by way of reimbursement from Medicare Australia. One of the eligibility criteria for HMRs is recent hospital discharge [16] and the HMR service has been demonstrated to be feasible as a post-discharge service for patients following ACS in the Australian healthcare setting [17]. However, the effect of this service on clinical outcomes following ACS is unknown.

\section{Justification for this trial}

Programs allowing pharmacists to provide formal medication review services exist across many countries. Although there are some regional differences in the structure of these programs, they are generally designed to improve the quality use of medicines and minimize the potential for medication-related harm. Previous studies assessing patient outcomes following post-discharge medication review services have found conflicting results. The HOMER trial questioned the value of the service as the intervention group had an increased hospitalization rate at six months post-discharge [18]. Similarly, Barker et al. trialed a home-based, post-discharge medication review service in patients with congestive heart failure (CHF) and found that patients in the intervention group had significantly longer $\mathrm{CHF}$ hospital stays, incidence rate ratio $=2.34(P<0.001)$ [19]. There were no statistical differences in the other two primary outcomes of death and hospital readmission rates. Conversely, Stewart et al. were able to demonstrate both a short- and long-term benefit by reducing hospital readmissions and mortality, following a post-discharge service targeted at a population that was suspected to be at a high risk for readmission [20,21]. While the goal of reducing healthcare costs through fewer and shorter hospitalizations appears appropriate, this outcome may not be a suitable measure for a service focusing specifically on medication management. A review by Benbassat et al. questioned the validity of hospital readmissions as a marker for quality care and highlighted that the length of hospital stays, readmission rates, and death appear to be mostly predicted by unmodifiable causes, such as age, disease severity, and co-morbidity [22]. The authors concluded by highlighting the importance of improving other 7clinical outcome measures, such as adherence to guideline-based therapy and improving patient's selfmanagement abilities.

Although the interventions trialed in their studies included a significant focus on improving medication adherence, neither Holland et al. nor Barker et al. measured changes in this outcome. As such, both authors were left to speculate over this outcome and how it may have affected hospital readmission rates. Conversely, Stafford et al. conducted a prospective, non-randomized, controlled cohort study of a pharmacist-led service aimed at improving warfarin therapy post-discharge and, while there were no significant changes in readmission or death rates over the 90-day follow-up period, the intervention was associated with a reduced rate of adverse bleeding events from warfarin therapy, $5.3 \% \mathrm{vs}$. $14.7 \%(P=0.03)$ and increased persistence with therapy, 95.4\% vs. $83.6 \%(P=0.004)$ [23]. The pharmacists involved in this trial were HMR-accredited, but also received additional education, specific to the needs of patients taking warfarin [24]. This ability to focus the HMR service on specific patient groups has been proposed as an avenue for improvement of the HMR service and this warrants further attention through future research [15]. We have considered both of these aspects in the modification of the service planned for this trial and have outlined that the period following hospital discharge is an appropriate time to target ACS patients due to their risk for harm resulting from premature medication discontinuation [2,3]. Furthermore, we have developed a detailed education program to help pharmacists address ACS-specific issues and encourage patient behavior change by using a motivational approach to the HMR patient interview.

\section{Objectives}

In this study, we aim to investigate the effect of an adaptation of the currently available HMR service on guideline-based medication adherence and persistence following ACS. The service will be directed towards the needs of ACS patients by educating the accredited pharmacists involved about ACS-specific patient issues and through improving the continuum of care by providing consistent information in a structured HMR referral letter. As such, the trialed service will be termed a directed Home Medicines Review (dHMR). This is the first trial in a series of studies entitled 'Medication Reviews ReDirected (MedReDi)' which refers to the plan for future research in this area, following a similar theme of minor adjustments to the currently existing HMR service, so that patients with other specific illnesses can be targeted through new dHMR services. The overall intention will be to help patients become more familiar and competent with their medication management aspect of therapy - more simply, helping patients become 'MedReDi'.

For the purpose of this study, guideline-based ACS medication therapy refers to the four medication classes as supported by ACS guidelines [25-27]. These 
medication classes include: angiotensin-converting enzyme (ACE) inhibitors/angiotensin II receptor blockers (ARBs); antithrombotics (aspirin and/or clopidogrel/ prasugrel and/or warfarin); beta-blockers; and statins.

\section{Methods/Design \\ Overview}

We will conduct a randomized controlled trial comparing the dHMR service delivered at two months post-discharge to usual care following an ACS admission. Changes in the pharmacist referral process and an ACSspecific education package to be completed by the study pharmacists make this program different to the standard HMR service. The primary outcome will be the proportion of patients who are adherent to a complete, guideline-based ACS medication regimen at six and twelve months post-discharge. Patients will be enrolled in hospital during an admission for ACS. Figure 1 provides an overview of the trial protocol. The design of the intervention and monitoring system has been based on a conceptual framework for the standardized evaluation of chronic disease management interventions, as developed by Lemmens et al. [28]. Figure 2 is a summary of this framework and Figure 3 is a comparison of how the components of this trial compare against the Lemmens et al. framework.

This representation of the 'Evaluation model for disease-management programs' has been adjusted slightly from the originally produced model to better reflect the points of the framework that we consider relevant to the HMR service (shown in further detail in Figure 3). It is important to recognize that this framework highlights the importance of considering patient-related factors, professional-related factors, and health-system factors in both the design and evaluation of interventions targeted toward improving the management of chronic diseases. These considerations have been particularly important throughout the development of this trial

\section{Setting}

Patient enrolment and dHMR referral will be from the two major tertiary referral hospitals in Tasmania, Australia. The Royal Hobart Hospital (RHH) is a 490-bed hospital with an 8-bed coronary care unit (CCU), percutaneous coronary intervention (PCI), and coronary artery bypass grafting (CABG) facilities, and accepts patients from across the state for CABG referrals [29]. The Launceston General Hospital (LGH) is a 300-bed hospital with a 4-bed coronary care unit and PCI facilities [30]. Both hospitals run cardiac rehabilitation programs that are offered to all ACS patients. Patients admitted to these centers generally receive verbal and written education regarding lifestyle changes following
ACS from nurses, and medication counseling from nurses or pharmacists, prior to discharge.

\section{Inclusion/Exclusion criteria}

All patients aged 18 to 80 years with a primary diagnosis of ACS presenting at the RHH and LGH will be considered for enrolment into the trial. The wide age range has been chosen to detect differences in the discharge management of patients across different age groups and to examine the suitability of the dHMR intervention across different age groups. The former problem has been previously recognized as an area for further interventional focus by Alexander et al. in the CRUSADE trial [31] and the latter has been recognized as an area requiring further investigation through a qualitative review of the HMR service [15].

Patients will be excluded from trial enrolment if they: are not returning to their home following hospital discharge (as this is a requirement of the existing HMR service); are non-Medicare eligible (for example, not a permanent Australian resident); have been diagnosed with a level of cognitive impairment such that the process of informed consent may be obscured; or have been diagnosed with a malignancy that is expected to be terminal within 12 months.

\section{Randomization}

We will use a computer-generated random sequence to provide the randomization coding. Patients will be randomized to the control or intervention groups following computerized recognition that the patient meets the inclusion criteria and has consented to trial involvement. The correct entry of this information into the central trial database will then enable the enrolling researcher to unlock the randomization status for that participant.

\section{Controls}

Following randomization to the control group, patients will be offered the usual care processes involved with post-discharge management of ACS in Australia. Through the public health system, patients are able to attend a cardiac rehabilitation program at their local hospital as well as a cardiologist follow-up appointment, usually occurring at one month following discharge. The cardiac rehabilitation programs from the two different hospitals may vary slightly in their level of physical activity, but the material covered in their information sessions is similar.

\section{Intervention}

Patients in the intervention group will receive a dHMR at approximately two months following discharge as well as usual care. The time of two months post- 


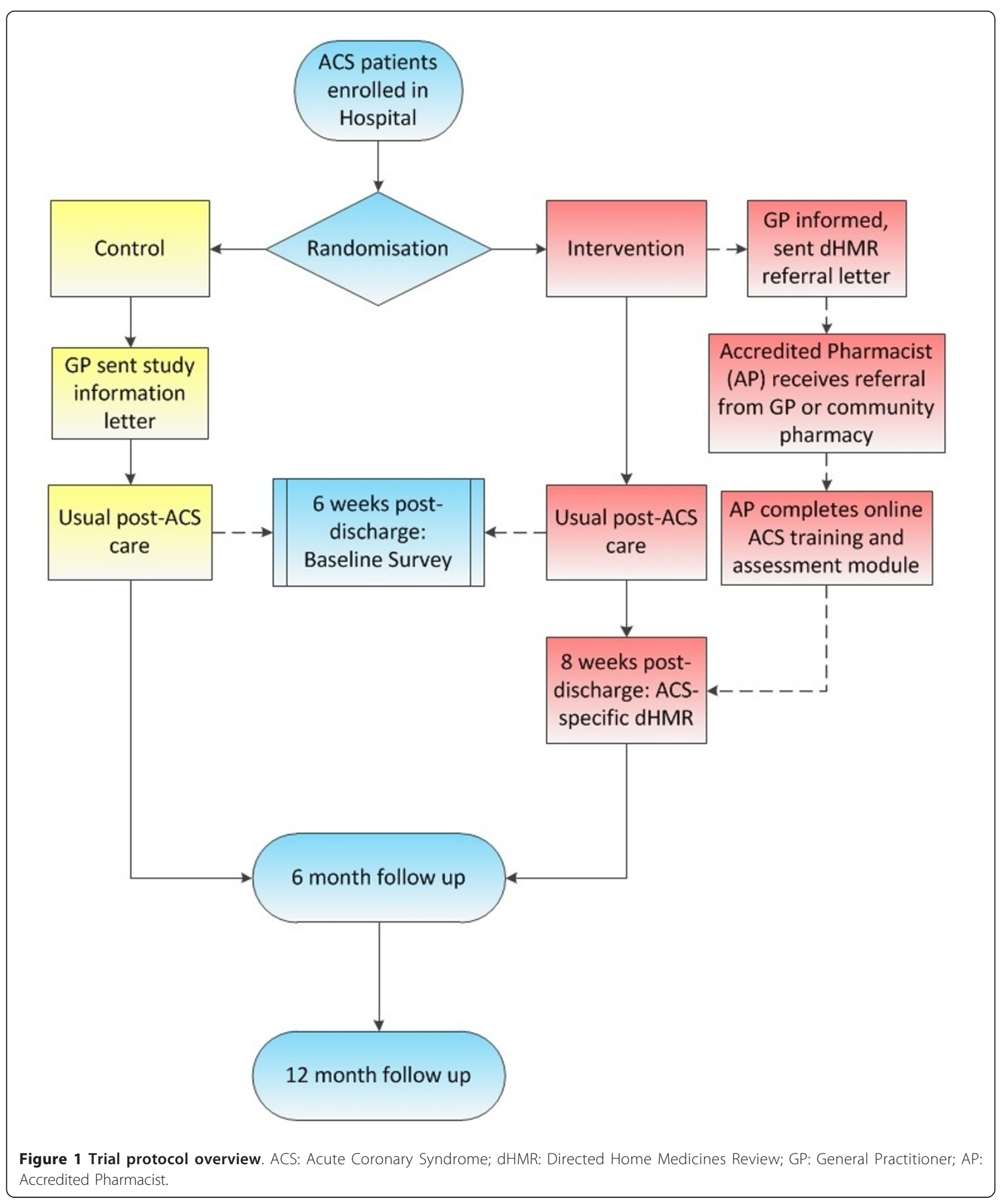

discharge was selected as previous studies of ACS patients have demonstrated that the period between one and three months post discharge is when patients are most vulnerable for medication discontinuation
$[2,3,32]$. This suggests that a service addressing both the educational and clinical management aspects of therapy may be most beneficial when offered in this time period. 

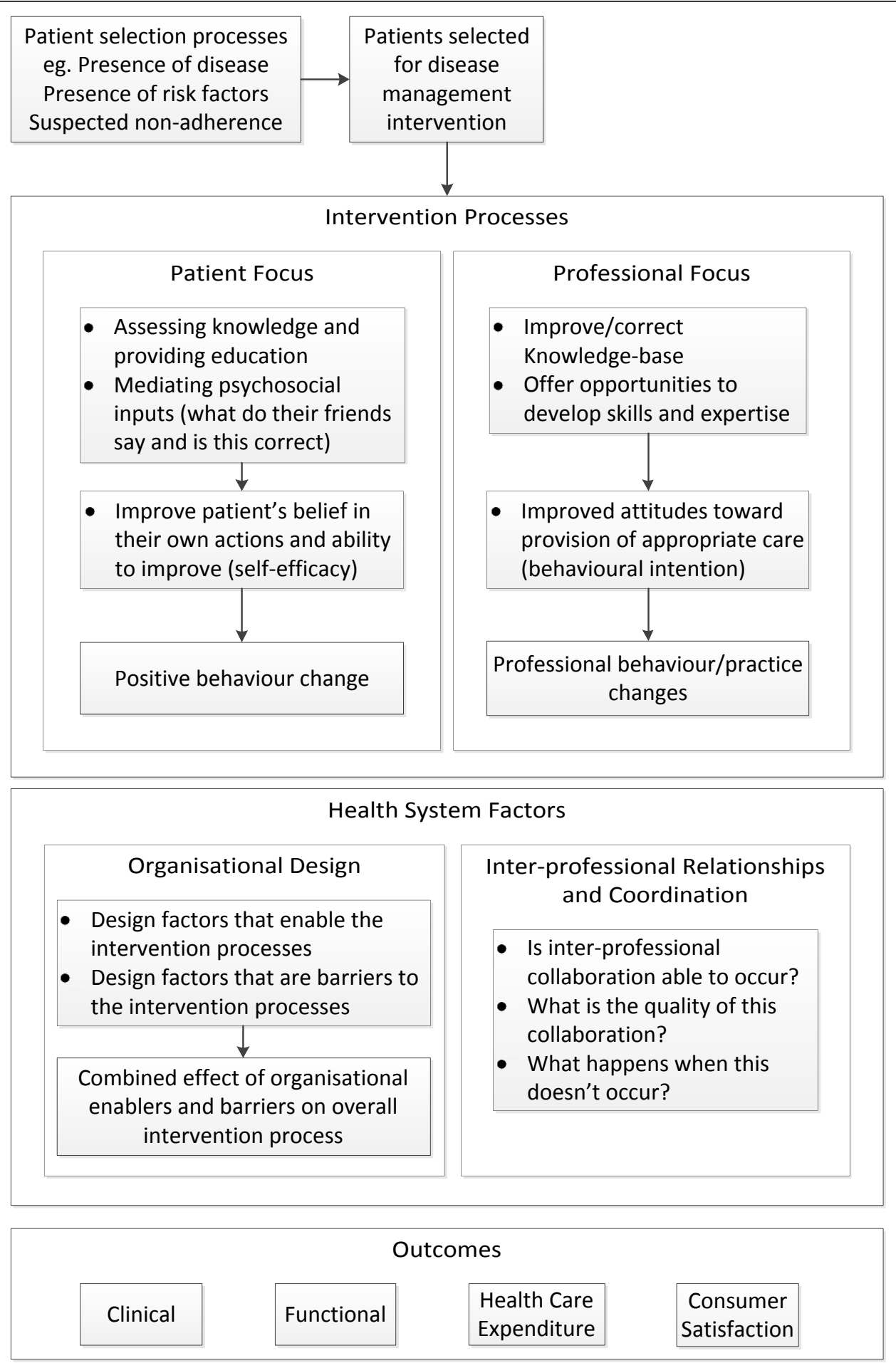

Figure 2 Summary of the Lemmens et al. framework [28]. This representation of the 'Evaluation model for disease-management programs' has been adjusted slightly from the originally produced model to better reflect the points of the framework that we consider relevant to the HMR service (shown in further detail through Figure 3). It is important to recognize that this framework highlights the importance of considering patient-related factors, professional-related factors, and health-system factors in both the design and evaluation of interventions targeted toward improving the management of chronic diseases. These considerations have been particularly important throughout the development of this trial. 

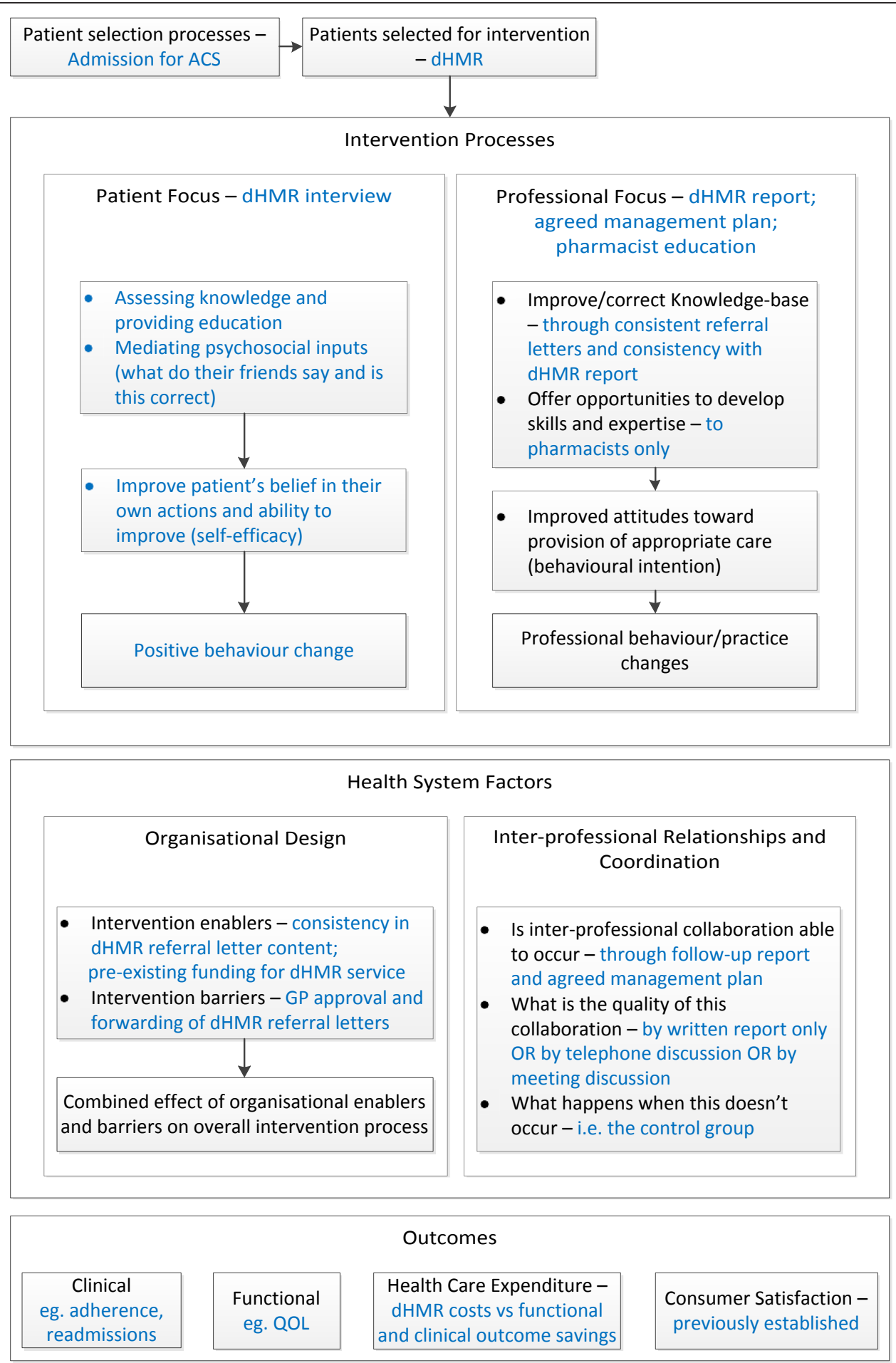

Figure 3 How the proposed dHMR service addresses the specific components of the Lemmens et al. framework [28]. ACS: Acute Coronary Syndrome; dHMR: Directed Home Medicines Review; GP: General Practitioner; QOL: Quality Of Life. The blue text highlights the areas of the framework where we are investigating the effect of a dHMR with specific detail provided for clarification.

Based on the existing funding arrangements and recommended reasons for referral, the HMR service is currently available in Australia for all ACS patients following hospital discharge [16]. The uptake of this service, however, may be largely limited by the absence of HMR referral systems in this transitional period. 
Furthermore, the currently available service could be tailored to maximize the potential for benefit from this service in the ACS population. We propose to add to the existing HMR service in several ways, making the proposed dHMR service more directed toward the ACS population.

Typically, HMRs are ordered by a referral letter generated by the patient's GP. Referral letters may vary significantly in their level of detail, potentially leaving the pharmacist with little direction prior to the patient interview. This concern was highlighted as a potential downfall of the service in a qualitative review that was conducted in 2008 [15]. To address this issue, the enrolling researchers will use a specifically designed database that allows baseline data collection to automatically populate sections of the HMR referral. The referral letter will still be sent to the GP for approval and addition of any further information, such as medications that have changed since discharge. GP approval is a requirement for payment under the existing public service arrangements, and having the GP engaged with the intervention early should increase their willingness to participate. This new approach, however, relieves the GP from having to complete most of the referral data and allows for simple addition of only useful information. The GP may also find the information on this referral letter useful when they receive the letter, as previous research has demonstrated that standard discharge letters following ACS admissions do not always contain a sufficient level of information and sometimes take too long to reach the GP [3].

In addition to the specific referral letter, the service will be further directed by offering accredited pharmacists across Tasmania an online education and assessment package. In order for a pharmacist to complete an interventional dHMR according to the trial protocol, they will be required to have a current accreditation status and have successfully completed the education and assessment package. Upon completion of the education and assessment package, accredited pharmacists will gain recognition of continuing professional development and an AUD\$50 reimbursement for their time.

\section{Developing the education and assessment package}

Within our trial, accredited pharmacists are appropriately poised to deliver education to patients at the HMR interview and to GPs through the HMR reports. Addressing both the patient and their GP in this fashion has been recognized as an important factor to increase the chance of improving patient outcomes through chronic disease management interventions [28,33,34]. Further education and specialization of accredited pharmacists in the area of ACS secondary prevention should increase the chance of the current service utilizing these opportunities for follow-on education.

The accredited pharmacist education and assessment package will consist of five online lectures, as well as several separate resource documents for pharmacists to refer to at any point throughout the HMR service. The five online lecture topics are: an introduction to the trial and the evidence to practice gaps for ACS; background information about the hospital management of ACS; the importance of cardiac rehabilitation and lifestyle modifications; evidence-based ACS medication management; and methods for improving adherence to therapy following ACS. The separate resource documents include a 'chest pain action plan', information on the use of shortacting nitrates, and links and referral to specific material available from the National Heart Foundation, Australia. The material will be assessed using case-based examples with multiple-choice questions (pass mark of 75\%). The full details of the development of the education and assessment package will be published at a later stage.

\section{Outcomes}

All outcomes will be measured using blinded assessment processes at the two study endpoints of six and twelve months post-discharge. Each outcome will be a comparison between the control and intervention groups. A proposed conceptual framework for the standardized evaluation of chronic disease management interventions has been considered in the selection of the trial outcomes and further trial evaluation [28]. Using a framework to guide outcome selection has not only helped to ensure that we are measuring the important clinical outcomes from the intervention, but also highlighted the individual steps of the intervention that require monitoring. Monitoring these individual steps, such as pharmacist detection of drug-related problems and GP acceptance of pharmacist recommendations, is important to measure the level of alignment between the theoretical plan and the practical application of the intervention. Such detailed monitoring and outcome reporting may also elucidate areas of practice worthy of further focus in future research.

\section{Primary outcome}

The primary outcome will be the proportion of patients who are adherent to a complete, guideline-based ACS medication regimen at the trial endpoints. The accepted definition of adherence for this trial will be a medication possession ratio (MPR) of $80 \%$ to $120 \%$ for all guidelinebased medications, which is a commonly accepted cutoff for this measure [35-39]. MPR will be determined by dividing the total number of days of medication supplied by the total specified time period. This value can be 
multiplied by 100 in order to be reported as a percentage of the time that the patient had medication available to take, a commonly used surrogate measure of adherence. During the baseline interview, we intend to record all possible pharmacies that a patient may attend in order to make the dispensing records complete. If, however, there is a significant shortfall in the completeness of dispensing records, we will use self-report of medication adherence as an alternate measure. The four-item Morisky adherence questionnaire will be used for this purpose [40]. This is a well-validated instrument, being used previously with good predictive validity in patients at risk of cardiovascular disease [40,41]. In the case where a patient has a clearly documented contraindication to one of the four guideline-based medication classes, their medication regimen may still be considered 'complete' provided they are taking all other guideline-based medications. For example, a patient with asthma not taking a beta-blocker but still taking aspirin, an ACE inhibitor, and a statin, will be considered in the same group as those prescribed all four medications. This primary outcome comprehensively assesses the overall impact of the intervention on both GP prescribing behaviors and patient medicationtaking behaviors. In order to separately measure the recognized problem of persistence with guideline-based medications, we will complete a secondary analysis of the primary outcome in the group of patients who are prescribed a complete, guideline-based medication regimen at the time of discharge from hospital.

\section{Secondary outcomes}

Secondary outcomes will include hospital readmission rates, length of hospital stays, changes in quality of life, cardiac rehabilitation completion rates, smoking cessations rates, and mortality. Hospital readmissions and mortality will be further categorized as ACS-related or due to other causes. Quality of life will be measured at six weeks postdischarge as a baseline, pre-dHMR measure and again at both study endpoints. The Euroqol 'EQ-5D 3 L' [42] will be used to measure general quality of life and the Seattle Angina Questionnaire (SAQ) [43] will be used to measure cardiac-specific quality of life. Both instruments have been validated when administered individually [43-46] and in combination [47] to assess quality of life in patients with coronary heart disease. In addition to a self-report of smoking status at baseline and the study endpoints, we will be further categorizing each smoker's dependence on cigarette smoking using the Fagerstrom Test for Nicotine Dependence (FTND) [48].

\section{Process outcomes}

In addition to reporting the important clinical outcomes that may be affected by the intervention, it is also recognized that there are many individual components of such interventions that can be measured and may highlight barriers or enablers to the overall success of the intervention [28]. From the professional side of the $\mathrm{dHMR}$, it will be important to measure GP acceptance of the intervention through approval of dHMR referrals, pharmacists' recognition of drug-related problems, the clinical relevance of the drug-related problems identified, and GP acceptance of pharmacists' recommendations. Figures 4 and 5 represent our plans to measure the effect of these professional and organizational barriers on trial implementation.

From the patient's perspective it is important to consider, firstly, how the dHMR process may influence psychological variables that have been previously recognized as relevant to adherence behavior, as well as how strongly these variables correlate to the outcome of adherence within this particular trial setting. The concepts we have chosen to consider and the validated instruments that will be used to evaluate each construct are detailed in Figure 6.

The concepts of knowledge [55-57], illness perception $[49,58]$, beliefs about medications $[59,60]$, and self-efficacy [50,61] have all been studied separately in coronary heart disease populations. In developing the conceptual framework for the evaluation of chronic disease interventions, however, Lemmens et al. recognized the important interrelations between these concepts and how this can affect the ultimate outcome of patient behavior change [28]. Risk perception [51] and adherence-specific behaviors [52] are two lesser studied concepts that may also affect or predict adherence and, as such, have been added to our model of assessment. As discussed, we consider it important to measure and report on the effect of the dHMR on these individual concepts, as well as the validity of the individual concepts as predictors for adherence within this trial setting. Again, the latter has been investigated on an individual basis for some concepts, but the different trial setting and the inclusion of further variables may result in alternate outcomes. The full details of the predictors of adherence recognized in our ACS population will be published elsewhere. The following is a brief summary of the questionnaires selected for this purpose: to assess medication knowledge we have selected recall of individual medication purpose as adapted from Hope et al. [53]; for illness perception we have selected the eight quantitative items of the Brief Illness Perception Questionnaire (Brief IPQ) [49]; for risk perception we have selected the Perceived Heart Risk Questionnaire (PHRQ) [51]; for beliefs about medications we have selected eight items from the Beliefs About Medicines Questionnaire (BMQ) [54] and developed two items to assess the impact of cost 


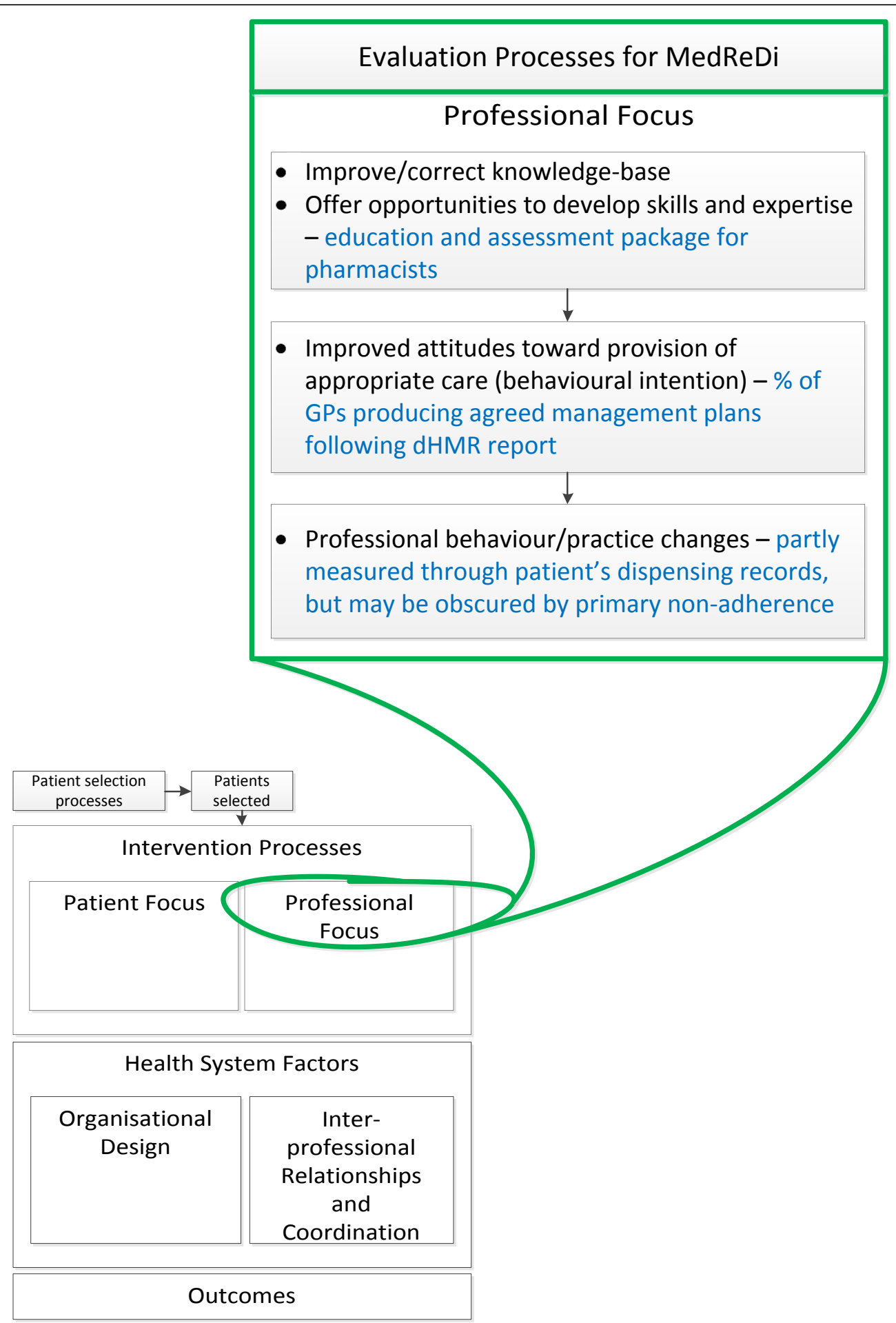

Figure 4 Evaluation of the professional-focused components of the intervention [28]. The blue text in this figure highlights where the professional focused components of the intervention fit into the Lemmens et al. framework. dHMR: Directed Home Medicines Review.

'The cost of my medications makes it difficult for me to take them regularly' and 'Medications are not good value for money' which will be assessed on the same 5-point Likert scale as the BMQ questions; for self- efficacy we have selected seven items from the Cardiac Self-Efficacy Scale [50]; for adherence-specific behaviors we have selected the Tool for Adherence Behavior Screening (TABS) [52]. 


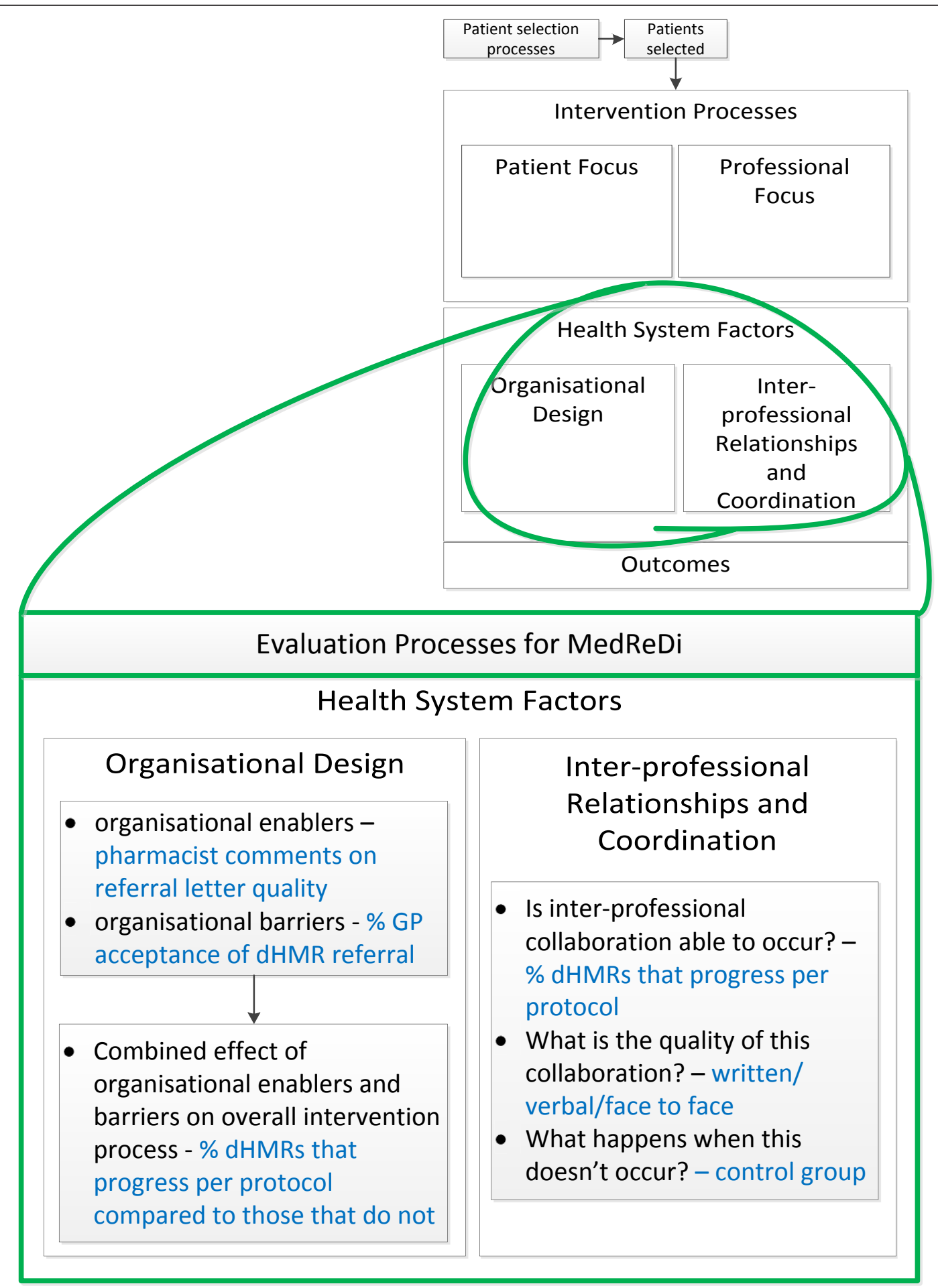

Figure 5 Evaluation of the health system's impact on the implementation of the intervention [28]. The blue text in this figure highlights how the local health-system structure may affect the implementation of the intervention described within this trial protocol. Again, the relevant points raised by the Lemmens et al. framework have been considered. dHMR: Directed Home Medicines Review.

Further detail on the reasoning behind each questionnaire's selection and the reasons for abbreviation of full questionnaires, where relevant, will be discussed in a future publication on the predictors of adherence. All of these questionnaires have been validated in populations of patients with coronary heart disease. 


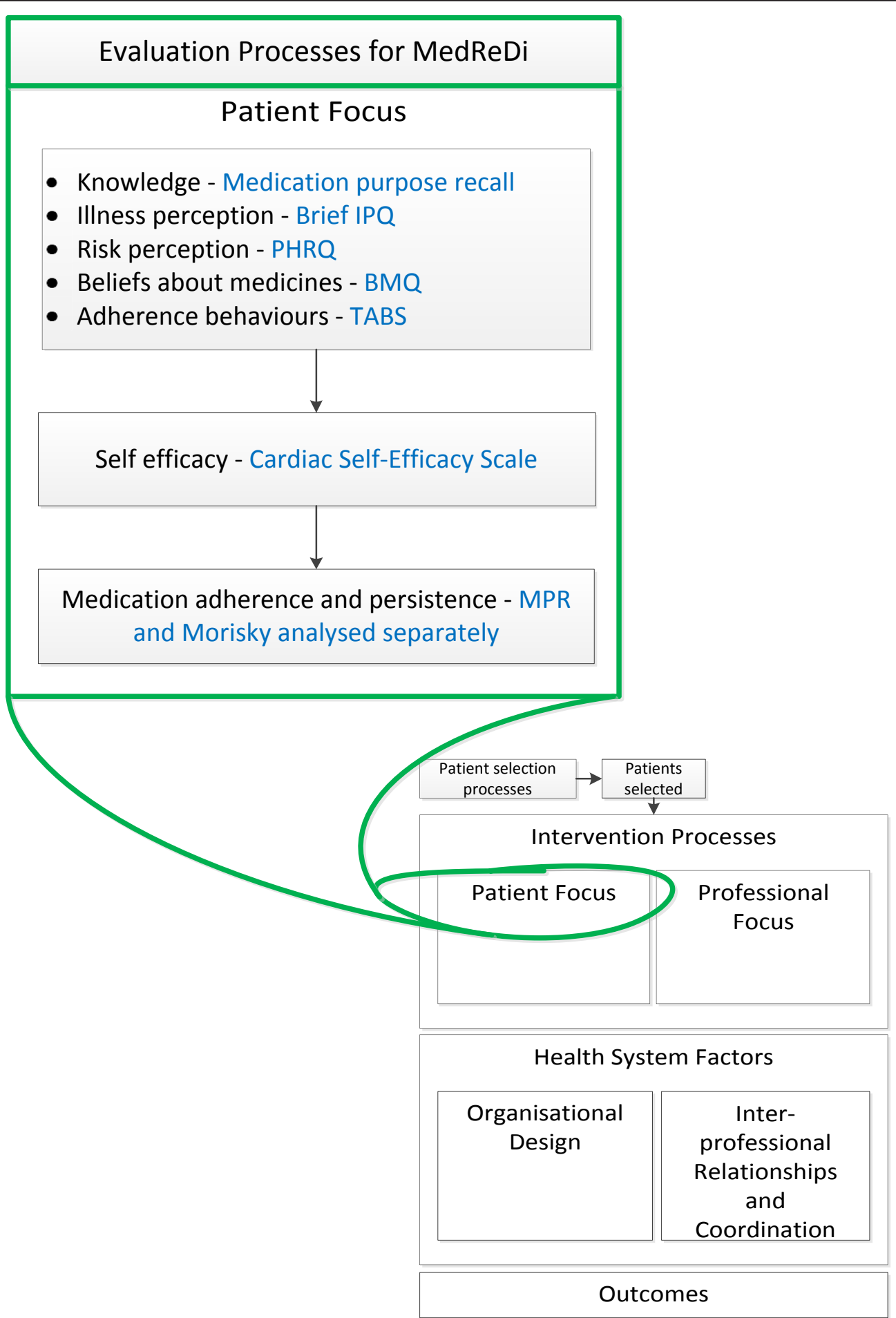

Figure 6 Evaluation of the patient focused component of the intervention [28,40,49-54]. This figure highlights the comprehensive evaluation that we have designed for the patient-focused component of the intervention. IPQ: Illness Perception Questionnaire; PHRQ: Perceived Health Risk Questionnaire; BMQ: Beliefs About Medicines Questionnaire; TABS: Tool for Adherence Behavior Screening; MPR: Medication Possession Ratio. 
In addition to these questionnaires it is important to note that for the purpose of testing for predictors of adherence, we will be considering two separate models. In the first model, adherence will be measured by the MPR and in the second model, adherence will be measured by the Morisky Adherence Questionnaire [40]. Both measures have individual benefits and limitations to their use, but have been extensively validated in cardiac settings $[35,40,41,62,63]$. It was considered important to include the second model using a questionnaire to assess adherence in order to maintain consistency with the reliance on questionnaires as the method of assessment for all concepts. The complete questionnaire set chosen for this study can be viewed in Additional file 1.

\section{Sample size}

We are aiming to detect a change in the primary outcome of $15 \%$ between the control and intervention groups. This predicted change is based on the results of the DMACS study, whereby a $12 \%$ post-intervention improvement was observed on the proportion of patients taking the same four guideline-based medications at discharge [3]. To detect a change of $15 \%$, assuming a control group result of $45 \%$, a power to detect a difference of $80 \%$ with alpha $=0.05$, we require a minimum sample size of 186 patients per group. To account for an approximate dropout rate of $20 \%$, we aim to enroll 465 patients in total.

\section{Data collection}

Baseline data collection will occur in hospital at the time of enrolment, immediately post-discharge from hospital and at six weeks post-discharge - when the participants will be sent their first questionnaire set. The in-hospital baseline data collection will include the recording of traditional coronary risk factors, as well as the 'cholesterol' version of the INTERHEART Modifiable Risk Score (IHMRS) [64]. This will allow for baseline comparison of risk between the two groups. Although not validated to calculate risk in a population of patients following a coronary event, the IHMRS requires the recording of risk factors in a way which emphasizes their correlation to their level of actual risk and this remains relevant, regardless of the patient's degree of heart disease. For example, the IHMRS requires the recording of smoking status into several cutoffs depending on the number of cigarettes smoked per day, which correlates independently to heart attack risk, as opposed to simply recording cigarette smoking status in an arbitrary fashion, which is of lesser value with regard to risk comparisons. The final baseline data collection point of six weeks post-discharge for questionnaire mail-out reflects our intention to gauge each patient's status on each of the questionnaires in the time directly before the HMR and not while in hospital, as factors, such as adherence behavior, may be very high in hospital but may significantly decline over time following discharge $[2,3,14,65,66]$. Patients in the intervention group will be asked to ensure that they complete their questionnaire before their pharmacist visit and pharmacists will record whether or not this has happened as reported by the patient. Patients in the control group will be given a reminder telephone call if their questionnaires have not been returned by three weeks from the mail-out date.

Accredited pharmacists' successful completion of the education and assessment package will be required prior to starting any trial dHMR. This will be recorded through an online system, specifically designed to guide each pharmacist through to completion of this package, while also monitoring their usage levels of the education website via separate log in codes. Although completion of the package is encouraged by an AUD\$50 honorarium, program completion cannot be enforced beyond this level and those patients receiving a dHMR by a pharmacist who has not successfully finished the education and assessment package prior to the dHMR interview will be excluded from the secondary on-treatment analysis, as discussed in the 'statistical analysis' section.

Following the dHMR interview, data collection starts with the pharmacist's dHMR report and the agreed, patient-GP management plan. Collection of these documents will allow for assessment of pharmacists' recognition of drug-related problems, the recommendations made to improve these problems, and the GPs' acceptance of these recommendations, as described in Figures 4 and 5. Telephone follow-up will be considered two months after the interview if these reports and management plans have not been received. Recent amendments to the Medicare-funded HMR reimbursement process have strongly increased the requirement that an agreed management plan be formulated following all HMRs, which should help facilitate this data collection point.

Six and twelve months post-discharge follow-up will include requests to community pharmacies for patient dispensing records, hospital register checks for readmissions and length of stays, a death's registry check for mortality, and questionnaire mail-out with a three-week phone call reminder.

\section{Statistical analysis}

All baseline variables will be compared between the control and intervention groups using independent samples t-tests for continuous variables and chi-squared analysis for categorical variables. All outcomes will be reported as descriptive comparisons between the control and intervention groups, with significance reported at alpha $=0.05$. Where primary outcomes reach statistical 
significance, any noted difference in the baseline group comparison will be tested by univariate comparison with stepwise addition into a multivariate model for those with univariate significance of $P<0.1$. Changes in each group's questionnaire results between baseline and study endpoints, as well as differences between intervention and control group's questionnaire results, will be reported. Further correlational investigations between questionnaire results and adherence will be reported elsewhere. As there are several potential barriers that may prevent a patient randomized to the intervention group from receiving a dHMR according to the protocol, we will use both an intention-to-treat analysis as well as an on-treatment sensitivity analysis.

\section{Ethical approval}

This trial has received ethical approval through the Tasmanian Health and Medical Human Research and Ethics Committee. Approval number: H11821. All participating patients will provide informed consent.

\section{Discussion}

Recent evidence supports the current need for an intervention directed at improving the prescription of, and patients' adherence to, guideline-based therapy in the period following ACS hospital discharge [2,3]. This trial aims to achieve this outcome using an intervention that is based on only minor adjustments to a currently existing and funded service, such that any success derived from this trial may be easily translated into regular practice. The potential for providing directly applicable benefits to society by using this proposed method was recognized in a recent editorial by Davidson and Macdonald [67]. Should our intervention lead to an improvement in the primary outcome, the inherent sustainability of the intervention in a real world environment is one of its major strengths.

As mentioned through this paper, the DMACS study demonstrated significant improvements in prescribing at discharge, but these improvements were almost completely lost at three months post-discharge [3]. Therefore it could be proposed that, ideally, the intervention described within this trial would occur in conjunction with a similar quality improvement study. This would ensure that both the in-hospital and post-discharge aspects of management were addressed together, allowing for an even greater opportunity to demonstrate sustained improvements. As the specific benefits from a dHMR service directed at ACS patients are unknown, however, it is logical to trial this service first, before considering its position in conjunction with other interventions. Australia's National Prescribing Service offers kits to allow ongoing drug use evaluations similar to that of the DMACS study, therefore a trial combining an in-hospital intervention and a post-discharge dHMR may be feasible for future research, should the current trial lead to success worthy of this follow-up.

Although based on an existing, Medicare-funded service, the treatment protocol faces several potential barriers to success. Largely, this includes interprofessional barriers, such as GP approval of the dHMR referrals and GP acceptance of pharmacists' recommendations. The former point will be aided by the enrolling researcher contacting the GP once randomization has been revealed. Although GP approval of dHMR referrals may affect the uptake of the intervention in this trial, the use of a consistent and informative referral letter and a telephone conversation to support this letter, could prove to be advantageous over the current systems for initiating standard HMR referrals, which may not always be this well-structured. The other barrier of GPs not accepting pharmacists' recommendations has been raised due to previous research showing that GPs only action approximately $45 \%$ of pharmacist's recommendations following HMRs $[68,69]$. The educational material provided to accredited pharmacists completing this trial, however, should help guide pharmacists to make recommendations which will be relevant post-ACS and simple to implement. Furthermore, as the education package has been based on the latest evidence in ACS management, even if GPs do not accept all of the pharmacists' recommendations, they should still be gaining useful information from the dHMR reports. This may lead to improved ACS management, irrespective of the rate at which GPs accept pharmacists' individual recommendations.

Having the pharmacists complete the online training package may also be another barrier to the successful implementation of the trial protocol. The education and assessment package should take approximately three hours to complete and will be rewarded with recognition of continuing professional development and an AUD \$50 honorarium. We hope that these incentives and the easy-to-use format of the material will help facilitate the completion of the package. Through careful consideration of the potential barriers recognized above, the dHMR processes that we have developed have transformed these barriers into opportunities to improve the implementation of the intervention.

We have designed a trial to test the effect of minor changes to an existing, funded model of intervention to target the needs of a high-risk population. The conceptually derived intervention, based on the Lemmens et al. framework, accommodates funding and other healthsystem-related barriers which are critical for successful implementation. The intervention will be assessed by observing changes in medication adherence which is an outcome that appropriately matches the aim of the 
intervention. We have outlined the potential benefit from the application of this intervention on a worldwide scale, should the intervention demonstrate positive outcomes in the trial population of Australian ACS patients.

\section{Trial status}

The trial has not yet started recruiting. The expected start time is early 2012.

\section{Additional material}

Additional file 1: MedReDi Patient Questionnaire Set [40,49,55-59].

\begin{abstract}
Abbreviations
ACE inhibitor: Angiotensin-Converting Enzyme Inhibitors; ACS: Acute Coronary Syndromes; AP: Accredited Pharmacist; ARBs: Angiotensin II Receptor Blockers; BMQ: Beliefs About Medicines Questionnaire; Brief IPQ: Brief Illness Perception Questionnaire; CABG: Coronary Artery Bypass Graft; CCU: Coronary Care Unit; CR: Cardiac Rehabilitation; dHMR: Directed Home Medicines Review; DMACS: Discharge Management of Acute Coronary Syndrome; FTND: Fagerstrom Test for Nicotine Dependence; GP: General Practitioner; HMR: Home Medicines Review; IHMRS: INTERHEART Modifiable Risk Score; LGH: Launceston General Hospital; MPR: Medication Possession Ratio; PCl: Percutaneous Coronary Intervention; PHRQ: Perceived Health Risk Questionnaire; QOL: Quality Of Life; RHH: Royal Hobart Hospital; SAQ: Seattle Angina Questionnaire; TABS: Tool for Adherence Behavior Screening
\end{abstract}

\section{Acknowledgements}

This trial has been granted funding through the Institutional Research Grants Scheme (IRGS) which operates under the University of Tasmania. The IRGS have not contributed to the study design, nor do they hold any conflict of interest in the reporting of this trial. The School of Pharmacy at the University of Tasmania has also contributed funding toward this trial. We would like to thank and acknowledge Mr Peter Gee for his current and future input into the design of the trial database system.

\section{Author details}

'Unit for Medication Outcomes Research and Education (UMORE), School of Pharmacy, University of Tasmania (UTAS), Sandy Bay Campus, Tasmania 7001, Australia. ${ }^{2}$ Centre for Cardiovascular and Chronic Care, Faculty of Nursing, Midwifery and Health, University of Technology Sydney (UTS), Sydney 2007, Australia.

\section{Authors' contributions}

DDLB, LS, LREB, and GMP have contributed equally to the study design process. PMD has contributed to the study design, with particular input on study evaluation and questionnaire selection. RLC has contributed to the study design. DDLB drafted this manuscript. All authors have contributed to the revision of the manuscript and approved of the final copy.

\section{Authors' information}

Mr Daniel DL Bernal is a current PhD candidate at the School of Pharmacy, UTAS, Tasmania, Australia. Ms Leanne Stafford has submitted her PhD on the topic of post-discharge medication review services to improve the use of warfarin therapy and currently works as a lecturer at the School of Pharmacy, UTAS, Tasmania, Australia. Dr Luke RE Bereznicki is the Deputy Head of School and Senior lecturer at the School of Pharmacy, UTAS, Tasmania, Australia. Dr Ronald L Castelino is a research fellow and lecturer at the School of Pharmacy, UTAS, Tasmania, Australia. Prof Patricia M Davidson is the Director of the Centre for Cardiovascular and Chronic Care at the UTS, Sydney, Australia. Prof Gregory M Peterson is the Head of School of Pharmacy and Director for UMORE, UTAS, Tasmania, Australia.

\section{Competing interests}

All of the authors declare no conflict of interest in the outcomes or conduct of this research.

Received: 21 December 2011 Accepted: 2 April 2012

Published: 2 April 2012

\section{References}

1. Chew DP, Huynh LT, Liew D, Astley C, Soman A, Brieger D: Potential survival gains in the treatment of myocardial infarction. Heart 2009, 95:1844-1850.

2. Ho PM, Spertus JA, Masoudi FA, Reid KJ, Peterson ED, Magid DJ, Krumholz HM, Rumsfeld JS: Impact of medication therapy discontinuation on mortality after myocardial infarction. Arch Intern Med 2006, 166:1842-1847.

3. Peterson GM, Thompson A, Pulver LK, Robertson MB, Brieger D, Wai A, Tett SE, for the Dmacs Project Group: Management of acute coronary syndromes at hospital discharge: do targeted educational interventions improve practice quality? J Healthc Qual 2011, doi:10.1111/j.19451474.2011.00137.x.

4. Fox KAA, Steg PG, Eagle KA, Goodman SG, Anderson FA, Granger CB, Flather MD, Budaj A, Quill A, Gore JM, for the GRACE Investigators: Decline in rates of death and heart failure in acute coronary syndromes, 19992006. JAMA 2007, 297:1892-1900.

5. Airoldi F, Colombo A, Morici N, Latib A, Cosgrave J, Buellesfeld L, Bonizzoni E, Carlino M, Gerckens U, Godino C, Melzi G, Michev I, Montorfano M, Sangiorgi GM, Qasim A, Chieffo A, Briguori C, Grube E: Incidence and predictors of drug-eluting stent thrombosis during and after discontinuation of thienopyridine treatment. Circulation 2007, 116:745-754.

6. lakovou I, Schmidt T, Bonizzoni E, Ge L, Sangiorgi GM, Stankovic G, Airoldi F, Chieffo A, Montorfano M, Carlino M, Michev I, Corvaja N, Briguori C, Gerckens U, Grube E, Colombo A: Incidence, predictors, and outcome of thrombosis after successful implantation of drug-eluting stents. JAMA 2005, 293:2126-2130.

7. Pfisterer M, Brunner-La Rocca HP, Buser PT, Rickenbacher P, Hunziker P, Mueller C, Jeger R, Bader F, Osswald S, Kaiser C: Late clinical events after clopidogrel discontinuation may limit the benefit of drug-eluting stents: an observational study of drug-eluting versus bare-metal stents. J Am Coll Cardiol 2006, 48:2584-2591.

8. Balaguer-Malfagon JR, Pomar-Domingo F, Vilar-Herrero JV, Planas-del Viejo AM, Perez-Fernandez E: Stent thrombosis in the modern era: incidence, outcome and predictive factors. Rev Esp Cardiol 2006, 59:842-845

9. Chew DP, Amerena J, Coverdale S, Rankin J, Astley C, Brieger D: Current management of acute coronary syndromes in Australia: observations from the acute coronary syndromes prospective audit. Intern Med J 2007, 37:741-748.

10. U. S. Department of Health and Human Services: Cardiac rehabilitation (Structured abstract). Clinical Practice Guideline 1995, 17:1-202.

11. Cardiac rehabilitation: what works, what doesn't, and why. [http://www. theheart.org/article/1257713/print.do].

12. Davies P, Taylor F, Beswick A, Wise F, Moxham T, Rees K, Ebrahim S: Promoting patient uptake and adherence in cardiac rehabilitation. Cochrane Database Syst Rev 2010, doi:10.1002/14651858.CD14007131. pub14651852.

13. Briffa TG, Kinsman L, Maiorana AJ, Zecchin R, Redfern J, Davidson PM, Paull G, Nagle A, Denniss AR: An integrated and coordinated approach to preventing recurrent coronary heart disease events in Australia Policy statement from the Australian Cardiovascular Health and Rehabilitation Association. Med J Aust 2009, 190:683-686.

14. Jiang $X$, Sit JW, Wong TKS: A nurse-led cardiac rehabilitation programme improves health behaviours and cardiac physiological risk parameters: evidence from Chengdu, China. J Clin Nurs 2007, 16:1886-1897.

15. Home medicines review program: qualitative research project final report. [http://www.health.gov.au/internet/main/publishing.nsf/Content/ B2992EBF12BE7E1ECA2573D8007F91F3/\$File/HMR\%20Final\%20Report.pdf].

16. Emblen $G$, Miller E: Home medicines review. The how and why for GPs. Aust Fam Physician 2004, 33:49-51. 
17. Ellitt GR, Engblom E, Aslani P, Westerlund T, Chen TF: Drug related problems after discharge from an Australian teaching hospital. Pharm World Sci 2010, 32:622-630.

18. Holland R, Lenaghan E, Harvey I, Smith R, Shepstone L, Lipp A, Christou M, Evans D, Hand C: Does home based medication review keep older people out of hospital? The HOMER randomised controlled trial. BMJ 2005, 330:293.

19. Barker A, Barlis P, Berlowitz D, Page K, Jackson B, Lim WK: Pharmacist directed home medication reviews in patients with chronic heart failure: a randomised clinical trial. Int J Cardiol, 10.1016/J.ljcard.2011.02.034.

20. Stewart S, Horowitz JD: Home-based intervention in congestive heart failure: long-term implications on readmission and survival. Circulation 2002, 105:2861-2866

21. Stewart S, Vandenbroek AJ, Pearson S, Horowitz JD: Prolonged beneficial effects of a home-based intervention on unplanned readmissions and mortality among patients with congestive heart failure. Arch Intern Med $1999,159: 257-261$.

22. Benbassat J, Taragin M: Hospital readmissions as a measure of quality of health care: advantages and limitations. Arch Intern Med 2000, 160:1074-1081

23. Stafford L, Peterson GM, Bereznicki LR, Jackson $S L$, van Tienen EC, Angley MT, Bajorek BV, McLachlan AJ, Mullan JR, Misan GM, Gaetani L: Clinical outcomes of a collaborative, home-based postdischarge warfarin management service. Ann Pharmacother 2011, 45:325-334.

24. Stafford L, Peterson GM, Bereznicki LR, Jackson SL, van Tienen EC: Training Australian pharmacists for participation in a collaborative, home-based post-discharge warfarin management service. Pharm World Sci 2010, 32:637-642.

25. Anderson JL, Adams CD, Antman EM, Bridges CR, Califf RM, Casey DE Jr, Chavey WE II, Fesmire FM, Hochman JS, Levin TN, Lincoff AM, Peterson ED, Theroux P, Wenger NK, Wright RS, Smith SC Jr, Jacobs AK, Antman EM, Halperin JL, Hunt SA, Krumholz HM, Kushner FG, Lytle BW, Nishimura R, Ornato JP, Page RL, Riegel B: ACC/AHA 2007 Guidelines for the management of patients with unstable angina/non ST-elevation myocardial infarction executive summary: a report of the American College of Cardiology/American Heart Association Task Force on Practice Guidelines. J Am Coll Cardiol 2007, 50:652-726, doi:652.

26. Bassand J-P, Hamm CW, Ardissino D, Boersma E, Budaj A, FernándezAvilés F, Fox KAA, Hasdai D, Ohman EM, Wallentin L, Wijns W, Vahanian A, Camm J, De Caterina R, Dean V, Dickstein K, Filippatos G, Kristensen SD, Widimsky P, McGregor K, Sechtem U, Tendera M, Hellemans I, Gomez JLZ, Silber S, Funck-Brentano C, Andreotti F, Benzer W, Bertrand M, Betriu A, et al: Guidelines for the diagnosis and treatment of non-ST-segment elevation acute coronary syndromes. Eur Heart J 2007, 28:1598-1660.

27. Acute Coronary Syndromes Guidelines Working Group: Guidelines for the management of acute coronary syndromes 2006. Med J Aust 2006, 184: S9-S29.

28. Lemmens KMM, Nieboer AP, van Schayck CP, Asin JD, Huijsman R: A model to evaluate quality and effectiveness of disease management. Qual Saf Health Care 2008, 17:447-453.

29. Royal Hobart Hospital. [http://dhhs.tas.gov.au/service_information/ service_delivery_points/royal_hobart_hospital].

30. Launceston General Hospital. [http://dhhs.tas.gov.au/service_information/ service_delivery_points/launceston_general_hospital].

31. Alexander KP, Roe MT, Chen AY, Lytle BL, Pollack CV Jr, Foody JM, Boden WE, Smith SC Jr, Gibler WB, Ohman EM, Peterson ED: Evolution in cardiovascular care for elderly patients with non-ST-segment elevation acute coronary syndromes: results from the CRUSADE National Quality Improvement Initiative. J Am Coll Cardiol 2005, 46:1479-1487.

32. Lee HY, Cooke CE, Robertson TA: Use of secondary prevention drug therapy in patients with acute coronary syndrome after hospital discharge. J Manag Care Pharm 2008, 14:271-280.

33. Von Korff M, Gruman J, Schaefer J, Curry SJ, Wagner EH: Collaborative management of chronic illness. Ann Intern Med 1997, 127:1097-1102.

34. Vinicor F, Cohen SJ, Mazzuca SA, Moorman N, Wheeler M, Kuebler T, Swanson S, Ours P, Fineberg SE, Gordon EE, Duckworth W, Norton JA, Fineberg NS, Clark CM Jr: DIABEDS: a randomized trial of the effects of physician and/or patient education on diabetes patient outcomes. J Chronic Dis 1987, 40:345-356

35. Bramley TJ, Gerbino PP, Nightengale BS, Frech-Tamas F: Relationship of blood pressure control to adherence with antihypertensive monotherapy in 13 managed care organizations. J Manag Care Pharm 2006, 12:239-245.

36. Christensen DB, Williams B, Goldberg HI, Martin DP, Engelberg R, LoGerfo JP: Assessing compliance to antihypertensive medications using computer-based pharmacy records. Med Care 1997, 35:1164-1170.

37. Erickson S, Coombs J, Kirking D, Azimi A: Compliance from self-reported versus pharmacy claims data with metered-dose inhalers. Ann Pharmacother 2001, 35:997-1003.

38. Guenette L, Moisan J, Preville M, Boyer R: Measures of adherence based on self-report exhibited poor agreement with those based on pharmacy records. J Clin Epidemiol 2005, 58:924-933.

39. Hamilton R, Briceland L: Use of prescription-refill records to assess patient compliance. Am J Health Syst Pharm 1992, 49:1691-1696.

40. Morisky DE, Green LW, Levine DM: Concurrent and predictive validity of a self-reported measure of medication adherence. Med Care 1986, 24:67-74.

41. Nelson MR, Reid CM, Ryan P, Willson K, Yelland L: Self-reported adherence with medication and cardiovascular disease outcomes in the second Australian National Blood Pressure Study (ANBP2). Med J Aust 2006, 185:487-489.

42. Brazier J, Jones $N$, Kind P: Testing the validity of the Euroqol and comparing it with the SF-36 health survey questionnaire. Qual Life Res 1993, 2:169-180

43. Spertus JA, Winder JA, Dewhurst TA, Deyo RA, Prodzinski J, McDonell M, Fihn SD: Development and evaluation of the Seattle angina questionnaire: a new functional status measure for coronary artery disease. J Am Coll Cardiol 1995, 25:333-341.

44. Dougherty CM, Dewhurst T, Nichol WP, Spertus J: Comparison of three quality of life instruments in stable angina pectoris: Seattle Angina questionnaire, short form health survey (SF-36), and quality of life indexcardiac version III. J Clin Epidemiol 1998, 51:569-575.

45. Ellis JJ, Eagle KA, Kline-Rogers EM, Erickson SR: Validation of the EQ-5D in patients with a history of acute coronary syndrome. Curr Med Res Opin 2005, 21:1209-1216.

46. Kahyaoglu Sut $H$, Unsar $\mathrm{S}$ : Is EQ-5D a valid quality of life instrument in patients with acute coronary syndrome? Anadolu Kardiyol Derg 2011 11:156-162.

47. Oreopoulos A, Padwal R, McAlister FA, Ezekowitz J, Sharma AM, KalantarZadeh K, Fonarow GC, Norris CM: Association between obesity and health-related quality of life in patients with coronary artery disease. Int J Obes (Lond) 2010, 34:1434-1441.

48. Heatherton TF, Kozlowski LT, Frecker RC, Fagerstrom KO: The Fagerstrom test for nicotine dependence - a revision of the Fagerstrom tolerance questionnaire. Br J Addict 1991, 86:1119-1127.

49. Broadbent E, Petrie KJ, Main J, Weinman J: The brief illness perception questionnaire. J Psychosom Res 2006, 60:631-637.

50. Sullivan MD, LaCroix AZ, Russo J, Katon WJ: Self-efficacy and self-reported functional status in coronary heart disease: a six-month prospective study. Psychosom Med 1998, 60:473-478.

51. Davidson PM, Salamonson Y, Rolley J, Everett B, Fernandez R, Andrew S, Newton PJ, Frost S, Denniss R: Perception of cardiovascular risk following a percutaneous coronary intervention: a cross sectional study. Int I Nurs Stud 2011, 48:973-978.

52. George J, Mackinnon A, Kong DC, Stewart K: Development and validation of the Beliefs and Behaviour Questionnaire (BBQ). Patient Educ Couns 2006, 64:50-60

53. Hope C, Wu J, Tu W, Young J, Murray M: Association of medication adherence, knowledge, and skills with emergency department visits by adults 50 years or older with congestive heart failure. Am J Health Syst Pharm 2004, 61:2043-2049.

54. Horne R, Weinman J, Hankins M: The beliefs about medicines questionnaire: the development and evaluation of a new method for assessing the cognitive representation of medication. Psychol Health 1999, 14:1-24.

55. Alm-Roijer C, Fridlund B, Stagmo M, Erhardt L: Knowing your risk factors for coronary heart disease improves adherence to advice on lifestyle changes and medication. J Cardiovasc Nurs 2006, 21:E24-E31.

56. Ghisi GLD, Durieux A, Manfroi WC, Herdy AH, de Carvalho T, Andrade A, Benetti M: Construction and validation of the CADE-Q for patient education in cardiac rehabilitation programs. Ara Bras Cardiol 2010, 94:813-822. 
57. Thanavaro JL, Thanavaro S, Delicath T: Coronary heart disease knowledge tool for women. J Am Acad Nurse Pract 2010, 22:62-69.

58. Grace SL, Krepostman S, Brooks D, Arthur H, Scholey P, Suskin N, Jaglal S, Abramson BL, Stewart DE: Illness perceptions among cardiac patients: relation to depressive symptomatology and sex. J Psychosom Res 2005, 59:153-160.

59. Allen LaPointe NM, Ou F-S, Calvert SB, Melloni C, Stafford JA, Harding T, Peterson ED, Alexander KP: Association between patient beliefs and medication adherence following hospitalization for acute coronary syndrome. Am Heart J 2011, 161:855-863.

60. Khanderia U, Townsend KA, Erickson SR, Vlasnik J, Prager RL, Eagle KA: Medication adherence following coronary artery bypass graft surgery: assessment of beliefs and attitudes. Ann Pharmacother 2008, 42:192-199.

61. Sarkar U, Ali S, Whooley MA: Self-efficacy and health status in patients with coronary heart disease: findings from the heart and soul study. Psychosom Med 2007, 69:306-312.

62. Choo PW, Rand CS, Inui TS, Lee M-LT, Cain E, Cordeiro-Breault M, Canning C, Platt R: Validation of patient reports, automated pharmacy records, and pill counts with electronic monitoring of adherence to antihypertensive therapy. Med Care 1999, 37:846-857.

63. Lavsa SM, Holzworth A, Ansani NT: Selection of a validated scale for measuring medication adherence. J Am Pharm Assoc 2011, 51:90-94.

64. McGorrian C, Yusuf S, Islam S, Jung H, Rangarajan S, Avezum A, Prabhakaran D, Almahmeed W, Rumboldt Z, Budaj A, Dans AL, Gerstein HC, Teo K, Anand SS, Investigators obotl: Estimating modifiable coronary heart disease risk in multiple regions of the world: the INTERHEART modifiable risk score. Eur Heart J 2011, 32:581-589.

65. Sud A, Kline-Rogers EM, Eagle KA, Fang J, Armstrong DF, Rangarajan K, Otten RF, Stafkey-Mailey DR, Taylor SD, Erickson SR: Adherence to medications by patients after acute coronary syndromes. Ann Pharmacother 2005, 39:1792-1797.

66. Ali RC, Melloni C, Ou FS, Schmader K, Ohman EM, Roe MT, Peterson ED, Alexander KP: Age and persistent use of cardiovascular medication after acute coronary syndrome: results from medication applied and sustained over time. J Am Geriatr Soc 2009, 57:1990-1996.

67. Davidson PM, Macdonald PS: Interventions for modern times: complex, collaborative, and culturally appropriate. Circ Cardiovasc Qual Outcomes 2011, 4:584-586

68. Gilbert AL, Roughead EE, Beilby J, Mott K, Barratt JD: Collaborative medication management services: improving patient care. Med J Aust 2002, 177:189-192

69. Castelino RL, Bajorek BV, Chen TF: Retrospective evaluation of home medicines review by pharmacists in older Australian patients using the medication appropriateness index. Ann Pharmacother 2010, 44:1922-1929.

doi:10.1186/1745-6215-13-30

Cite this article as: Bernal et al: Home medicines reviews following acute coronary syndrome: study protocol for a randomized controlled trial. Trials 2012 13:30.

\section{Submit your next manuscript to BioMed Central and take full advantage of:}

- Convenient online submission

- Thorough peer review

- No space constraints or color figure charges

- Immediate publication on acceptance

- Inclusion in PubMed, CAS, Scopus and Google Scholar

- Research which is freely available for redistribution

Submit your manuscript at www.biomedcentral.com/submit
Biomed Central 